\title{
Adsorción de Cadmio en Solución utilizando como Adsorbente Material Tobáceo Modificado
}

\author{
Monica L. Rueda ${ }^{(1) *}$, Cristina Volzone ${ }^{(2)}$ y Susana Y. Martinez ${ }^{(1)}$ \\ (1) Asentamiento Universitario Zapala, Facultad de Ingeniería, Universidad Nacional del Comahue, \\ 12 de Julio y Rahue, (8340) Zapala, Neuquén, Argentina (e-mail: uncmonica zapala@hotmail.com) \\ (2) Centro de Tecnología de Recursos Minerales y Cerámica-CETMIC (CONICET La Plata/CIC), \\ C.C.49, Cno. Centenario y 506, (1897) M.B.Gonnet, Buenos Aires, Argentina.
}

*autor a quien debe ser dirigida la correspondencia

Recibido Sept. 07, 2009; Aceptado Nov. 20, 2009; Versión Final recibida Dic. 21, 2009

\begin{abstract}
Resumen
Se estudia la adsorción de iones de cadmio en solución por un mineral tobáceo, procedente de la Provincia de Neuquén, Argentina, utilizado en forma natural y luego de concentrado en fracción arcilla. El análisis químico fue efectuado por Plasma Acoplado Inductivamente y la composición mineralógica fue identificada por Difracción de Rayos $X$. La isoterma de adsorción fue obtenida usando cadmio en solución en el rango de concentración de 100 a 1000 ppm. El ensayo experimental se llevó a cabo con una relación sólido/liquido de $2 \%$ p/v y tiempo de contacto de 24 horas. La retención de cadmio se determinó por diferencia entre la concentración inicial y la concentración luego del contacto con el sólido, lectura efectuada por espectrometría de absorción atómica. La mayor proporción de fracción arcillosa (esmectita e illita) lograda por la purificación, permitió reducir el contenido de cuarzo y feldespato y aumentar la capacidad de adsorción de 15.75 a $22.86 \mathrm{mg} \cdot \mathrm{g}^{-1}$.
\end{abstract}

Palabras claves: adsorción, cadmio, material tobáceo, mineral arcilloso, purificación

\section{Adsorption of Cadmium in Solution using a Modified Tuffaceous Material as Adsorbent}

\begin{abstract}
The adsorption of $\mathrm{Cd}$ ions from solution by a tuffaceous material (in natural and after clay concentration) from Neuquén Province, Argentine was tested. The solid was used as collected and after being concentrated in the clay fraction. The chemical analysis was performed by Plasma Couple Inductively and the mineralogical composition was identified by X-ray diffraction. The adsorption isotherms were carried out by using concentrations of Cd solutions in the range of 100 to $1000 \mathrm{ppm}$. The solid/liquid ratio was $2 \% \mathrm{w} / \mathrm{v}$ and the contact time was 24 hours. The amount of metal ion retained was obtained by difference between the initial concentration and supernatant concentration after contacting metal ions solution with the solid. The metal concentrations were determined by atomic absorption spectrometry. The main mineralogical components of the sample were smectite/illite (clay minerals), quartz and feldspar. The purification of the tuffaceous material in clay minerals increased the adsorption capacity from 15.75 to $22.86 \mathrm{mg} \cdot \mathrm{g}^{-1}$.
\end{abstract}

Keywords: adsorption, cadmium, tuffaceous material, clay mineral, purification 


\section{INTRODUCCIÓN}

El vertido incontrolado de residuos urbanos y procedentes de distintas industrias, incluyendo las actividades mineras y agrícolas ha ocasionado serios problemas en los recursos hídricos, poniendo en peligro el equilibrio de diferentes ecosistemas. Existen distintas vías de movilización de las sustancias contaminantes, sin embargo el medio acuático tiene particular relevancia ya que éste permite el transporte por largas distancias de iones metálicos y distintos compuestos químicos. El problema de la contaminación de las aguas ha causado una mayor exigencia en el control del vertido de residuos peligrosos, además ha conducido a la implementación de normas más restrictivas respecto a los niveles de compuestos tóxicos y/o peligrosos permitidos en desechos.

Esta problemática ha interesado a la comunidad científica, quienes han estudiado posibles soluciones con el fin de mitigar los efectos perniciosos sobre el medioambiente. Dentro de las metodologías a implementar se puede citar el tratamiento de desechos con carbones activados, zeolitas naturales y sintéticas, minerales arcillosos, entre otros; por medio de adsorción. La adsorción consiste en el enriquecimiento de la interfase en una de las especies presentes en el sistema, en este caso los iones cadmio sobre la superficie del sólido. Este procedimiento puede ser empleado con diferentes objetivos tales como purificación de efluentes, recuperación de metales en solución y separación de componentes en una mezcla, entre otros. En condiciones experimentales controladas, un sustrato es capaz de atrapar selectivamente sobre su superficie iones disponibles en una fase acuosa envolvente, fenómeno que permite retener un ion metálico en particular.

Los materiales arcillosos han sido empleados para llevar a cabo experiencias de adsorción ya que poseen propiedades fisicoquímicas las cuales derivan de su tamaño de partícula inferior a $2 \mu \mathrm{m}$, morfología laminar y capacidad de cambiar fácilmente los iones fijados en la superficie exterior de sus cristales y en los espacios interlaminares, por otros existentes en soluciones acuosas, propiedad conocida como capacidad de intercambio catiónico, siendo las esmectitas los de mayor aplicación.

Las esmectitas presentan una estructura básica del tipo 2:1 o T-O-T, es decir formadas por una capa octaédrica que contiene iones aluminio y magnesio, situada entre dos capas tetraédricas de silicio. El reemplazo de silicio por aluminio en la capa tetraédrica y aluminio por hierro o magnesio en la octaédrica, origina un déficit de carga, equilibrada por cationes alcalinos o alcalinotérreos que se ubican en el espacio interlaminar (Newman et al.,1987; Sun Kou et al., 2004).

Diversas publicaciones científicas reportan la aplicación de tratamientos fisicoquímicos tales como, pilarización, activación ácida o alcalina, homoionización, entre otros, practicados con la finalidad de mejorar la capacidad de retención (Matthes et al., 1999; Grzegorz, 2002; Aşçı et al., 2007).

Una roca que posee cierto contenido de partículas inferiores a $2 \mu \mathrm{m}$, según su grado de alteración, es conocida con el nombre de toba. Esta roca es de origen piroclástico y se forma por acumulación de depósitos cineríticos durante episodios volcánicos, que por alteración diagenética en ambientes lagunares o lacustres originan minerales arcillosos (Mazzoni, 1986).

Si bien diferentes autores han utilizado como adsorbentes zeolitas, caolines, vermiculitas y arcillas para retener cadmio (Pavón et al., 2000; Coles y Yong, 2002; Vieira dos Santos y Masini, 2007; AbuEishah, 2008), se seleccionó para el presente trabajo una toba, roca abundante en la naturaleza, de bajo costo y fácil extracción, a fin de evaluar su eficiencia para la remoción de cadmio en solución acuosa.

\section{PROCEDIMIENTO EXPERIMENTAL}

Un material tobáceo proveniente de la Formación Collón Cura, del Mioceno superior, perteneciente a la provincia de Neuquén, Argentina, fue utilizado como materia prima. El material fue molido pasante M:200 $(<74 \mu \mathrm{m})$ y denominado M1. Para evaluar la influencia de diferentes componentes mineralógicos sobre la retención de cadmio, una fracción por debajo de $2 \mu \mathrm{m}$ del mineral M1 fue obtenido por sedimentación, la cual fue denominada M1f. Los sólidos fueron caracterizados por Difracción de Rayos X en un equipo Philips PW 3020 con tubo de cobre a $40 \mathrm{kV}$ y $20 \mathrm{~mA}$. Los análisis químicos se realizaron empleando Plasma Acoplado Inductivamente, bajo norma ISO 9002. 
Diferentes concentraciones de cadmio en solución acuosa fueron preparadas para evaluar la capacidad de adsorción de los materiales tobáceos. Los ensayos de adsorción se practicaron empleando una solución Standard Titrisol Merck ${ }^{\circledR}$ de cadmio, conteniendo $1000 \mathrm{mg} \cdot \mathrm{L}^{-1}(\mathrm{ppm}) \mathrm{del}$ catión. Las disoluciones fueron de 1000, 750, 500, 250 y $100 \mathrm{mg} \cdot \mathrm{L}^{-1}$. Los ensayos de adsorción se realizaron con una relación sólido/líquido del $2 \% \mathrm{p} / \mathrm{v}$, durante 24 horas, separando posteriormente los sólidos por centrifugación.

La concentración de la solución luego de la adsorción fue medida por Espectrometría de Absorción Atómica con un equipo Ati Unicam 929 AASpectrometer.

\section{RESULTADOS Y DISCUSIONES}

La Fig. 1 muestra el espectro de Difracción de Rayos X para el material tobáceo M1. La composición mineralógica indica presencia de esmectita, illita, cuarzo y feldespato. El análisis por Difracción de Rayos $X$ de la fracción inferior a $2 \mu \mathrm{m}$ de la toba (M1f) muestra un incremento del contenido de esmectita e illita, es decir, las impurezas de cuarzo y feldespato fueron reducidas.

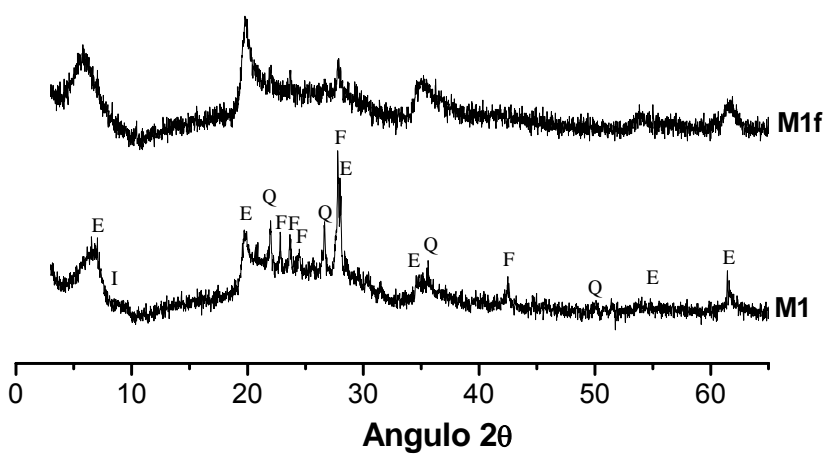

Fig. 1: Difracción de Rayos $X$ de la muestra M1 y M1f.

E: esmectita, I: illita, Q: cuarzo, F: feldespato.

La significativa disminución del contenido de sílice, componente del cuarzo; y del aluminio, calcio y potasio, componentes de los feldespatos, Tabla 1, se corresponden con el incremento en el material arcilloso de la muestra M1f, como lo indica el análisis por Difracción de Rayos X.

Tabla 1: Análisis Químicos

\begin{tabular}{|l|c|c|c|c|c|c|c|c|c|}
\hline MUESTRAS & $\begin{array}{c}\mathrm{SiO}_{2} \\
\%\end{array}$ & $\begin{array}{c}\mathrm{Al}_{2} \mathbf{O}_{3} \\
\%\end{array}$ & $\begin{array}{c}\mathrm{Fe}_{2} \mathbf{O}_{3} \\
\%\end{array}$ & $\begin{array}{c}\text { MgO } \\
\%\end{array}$ & $\begin{array}{c}\mathbf{C a O} \\
\%\end{array}$ & $\begin{array}{c}\mathbf{N a}_{2} \mathbf{O} \\
\%\end{array}$ & $\begin{array}{c}\mathbf{K}_{2} \mathbf{O} \\
\%\end{array}$ & $\begin{array}{c}\mathrm{TiO}_{2} \\
\%\end{array}$ & $\begin{array}{c}\mathbf{L O I} \\
\%\end{array}$ \\
\hline M1 & 62,19 & 12,93 & 4,86 & 0,99 & 1,92 & 2,69 & 1,37 & 0,66 & 12,00 \\
\hline M1f & 56,21 & 12,84 & 7,93 & 1,68 & 1,22 & 1,94 & 0,89 & 0,88 & 16,10 \\
\hline
\end{tabular}

La Fig. 2 muestra las isotermas de adsorción de cadmio, utilizando el material tobáceo en su estado natural y con posterior purificación en fracción arcilla (esmectita).

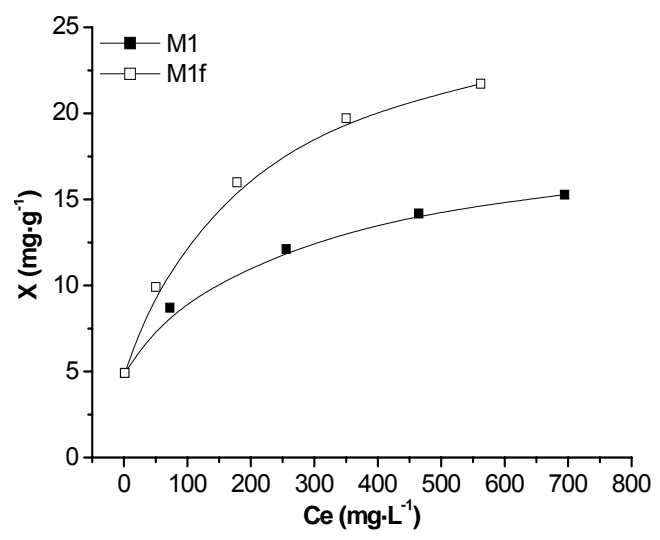

Fig. 2: Isotermas de Adsorción. 
Se observa una marcada diferencia en adsorción entre ambas muestras, siendo mayor cuando la toba ha sido purificada, es decir las impurezas fueron removidas y el contenido arcilloso (esmectita) es mayor. Los resultados ajustaron con la ecuación de Langmuir $\left(R^{2}: 0,98 ; 0,99\right)$, permitiendo obtener el valor de máxima adsorción de la monocapa para cada una de las muestras, correspondiendo valores de $15,75 \mathrm{mg} \mathrm{Cd} \mathrm{g}^{-1}$ y $23 \mathrm{mg} \mathrm{Cd} \mathrm{g}^{-1}$ para la toba natural M1 y enriquecida en arcilla M1f, respectivamente. Coles y Yong (2002), informaron valores aproximados de retención de cadmio de $2 \mathrm{mg} \cdot \mathrm{g}^{-1}$, utilizando caolinita como adsorbente en tanto que, Pavón et al. (2000), reportaron retenciones en el orden de $7-15 \mathrm{mg} \mathrm{Cd} \mathrm{g}^{-1}$, empleando sustratos zeolíticos.

\section{CONCLUSIONES}

La isoterma de adsorción del material tobáceo respondió al modelo matemático de Langmuir $\left(R^{2}=0,999\right)$. Asimismo, un comportamiento similar se observó al utilizar como sólido adsorbente la fracción rica en minerales arcillosos.

El material tobáceo enriquecido en partículas inferiores a $2 \mu \mathrm{m}$, fracción arcillosa, favoreció la retención de cadmio respecto a la muestra original en un $50 \%$, alcanzando un valor de $22,86 \mathrm{mg} \cdot \mathrm{g}^{-1}$.

\section{AGRADECIMIENTOS}

Los autores agradecen a las instituciones que hacen posible el desarrollo de esta investigación a través del proyecto FAIN No. 141, Universidad Nacional del Comahue (UNCo) y CONICET.

\section{REFERENCIAS}

Aşçı Y., Nurbaş M. y Sağ Açıkel Y., Sorption of Cd(II) onto kaolin as a soil component and desorption of $\mathrm{Cd}$ (II) from kaolin using rhamnolipid biosurfactant, Journal of Hazardous Materials, 139 (1), 50-56 (2007).

Coles, C.A. y R. N. Yong, Aspects of kaolinite characterization and retention of $\mathrm{Pb}$ and $\mathrm{Cd}$, Applied Clay Science: 22, 39-45 (2002).

Grzergorz, J., Effect of acid and alkali treatments on surface-charge properties of selected minerals, Clays and Clay Minerals: 50, 647-656 (2002).

Matthes, W., F. T. Madsen y G. Kahr, Sorption of heavy-metal cations by Al and Zr-hidroxyintercalated and pillared bentonite, Clays and Clay Minerals, 48, 617-629 (1999).

Mazzoni, M., Procesos y depósitos piroclásticos, $1^{\circ}$ edición, Asociación Geológica Argentina, Buenos Aires, Argentina (1986).

Newman, A.C.D., Chemistry of clays and clays, $1^{\circ}$ edición, 01-480. Longman Scientific \& Technical, London, England (1987).

Pavón, T. B., E. Campos y M. T. Olguín, Remoción de Níquel, Cadmio y Zinc del agua, utilizando clinoptilolita heulandita, Ciencia Ergo Sum: 7 (3), 251-258 (2000).

Samir I. Abu-Eishah, Removal of $\mathrm{Zn}, \mathrm{Cd}$, and $\mathrm{Pb}$ lons from water by Sarooj clay, Applied Clay Science: 42 (1-2), 201-205 (2008).

Sun-Kou, M. R., Volzone, C. y Sapag, K., Las arcillas y sus diferentes aplicaciones en adsorción, Adsorbentes en la solución de algunos problemas ambientales, $1^{\circ}$ edición, CYTED, 165-192, Madrid, España (2004).

Vieira dos Santos A.C. y Masini, J.C., Evaluating the removal of $\mathrm{Cd}(\mathrm{II}), \mathrm{Pb}(\mathrm{II})$ and $\mathrm{Cu}(\mathrm{II})$ from a wastewater sample of a coating industry by adsorption onto vermiculite, Applied Clay Science: $37,167-174$ (2007). 